\title{
Where has all the influenza gone? The impact of COVID-19 on the circulation of influenza and other respiratory viruses, Australia, March to September 2020
}

Sheena G Sullivan ${ }^{1}$, Sandra Carlson ${ }^{2}$, Allen C Cheng ${ }^{3,4}$, Monique BN Chilver ${ }^{5}$, Dominic E Dwyer ${ }^{6}$, Melissa Irwin ${ }^{7}$, Jen Kok ${ }^{6}$,

Kristine Macartney ${ }^{8,9}$, Jennifer MacLachlan ${ }^{10}$, Cara Minney-Smith ${ }^{11}$, David Smith ${ }^{11,12}$, Nigel Stocks ${ }^{5}$, Janette Taylor ${ }^{6}$, Ian G Barr ${ }^{13}$

1. WHO Collaborating Centre for Reference and Research on Influenza, Royal Melbourne Hospital, and Doherty Department, University of Melbourne, at the Peter Doherty Institute for Infection and Immunity, Melbourne, Australia

2. FluTracking, Hunter New England Population Health, Newcastle, Australia

3. School of Public Health and Preventive Medicine, Monash University, Melbourne, Australia

4. Department of Infectious Diseases, Alfred Health, and Central Clinical School, Monash University, Melbourne, Australia

5. Discipline of General Practice, University of Adelaide, Adelaide, Australia

6. Centre for Infectious Diseases and Microbiology Laboratory Services, New South Wales Health Pathology - Institute of Clinical Pathology and Medical Research, Westmead Hospital, Westmead, Australia

7. Rapid Surveillance, Centre for Epidemiology and Evidence, NSW Ministry of Health, Sydney, Australia

8. National Centre for Immunisation Research and Surveillance and The Children's Hospital Westmead, Sydney, Australia

9. Faculty of Medicine and Health, The University of Sydney, Sydney, Australia

10. WHO Collaborating Centre for Viral Hepatitis, Royal Melbourne Hospital at the Peter Doherty Institute for Infection and Immunity, Melbourne, Australia

11. PathWest Laboratory Medicine WA, Nedlands, Australia

12. Faculty of Health and Medical Sciences, University of Western Australia, Nedlands, Australia

13. WHO Collaborating Centre for Reference and Research on Influenza, Royal Melbourne Hospital, and Department of Microbiology and Immunology, University of Melbourne, at the Peter Doherty Institute for Infection and Immunity, Melbourne, Australia

Correspondence: Sheena Sullivan (sheena.sullivan@influenzacentre.org)

Citation style for this article:

Sullivan Sheena G, Carlson Sandra, Cheng Allen C, Chilver Monique BN, Dwyer Dominic E, Irwin Melissa, Kok Jen, Macartney Kristine, MacLachlan Jennifer, MinneySmith Cara, Smith David, Stocks Nigel, Taylor Janette, Barr Ian G. Where has all the influenza gone? The impact of COVID-19 on the circulation of influenza and other respiratory viruses, Australia, March to September 2020. Euro Surveill. 2020;25(47):pii=2001847.

Article submitted on 23 Oct 2020 / accepted on 26 Nov 2020 / published on 26 Nov 2020

The coronavirus disease pandemic was declared in March 2020, as the southern hemisphere's winter approached. Australia expected co-circulation of severe acute respiratory syndrome coronavirus 2 , influenza and other seasonal respiratory viruses. However, influenza notifications were 7,029 (MarchSeptember) compared with an average 149,832 for the same period in 2015-2019*, despite substantial testing. Restrictions on movement within and into Australia may have temporarily eliminated influenza. Other respiratory pathogens also showed remarkably changed activity in 2020.

The World Health Organization (WHO) declared a coronavirus disease (COVID-19) pandemic on 11 March 2020, as southern hemisphere countries prepared for their usual winter respiratory pathogen epidemics. The announcement prompted concern that severe acute respiratory syndrome coronavirus 2 (SARS-CoV-2), influenza and other respiratory viruses might co-circulate, straining and possibly overwhelming healthcare systems. In Australia, these fears were not realised and-in contrast to expectations-influenza activity was at an all-time low during the southern hemisphere's 2020 winter. Here, we describe the decreased activity of influenza and other respiratory pathogens in
Australia and the measures that have likely contributed to their decline.

\section{Pandemic mitigation measures and influenza and COVID-19 notifications}

Key government measures to restrict movement and mixing are displayed in Figure $1 \mathrm{~A}$, with severity indicated using the Oxford Stringency Index [1]. Like many other countries, COVID-19 mitigation measures implemented in Australia included working from home, limits on types of indoor and outdoor social gatherings (such as meals, organised sport and religious services) and numbers of attendees, visitor restrictions in hospitals and residential long-term care facilities, increased use of hand sanitisers and other hygiene measures, and physical distancing [2]. The duration and intensity of these measures varied nationwide, with Victoria the only jurisdiction to require persons (aged $>12$ years) to wear face masks in public and to carry out extended school closures. Domestic inter-jurisdictional borders were closed by five of eight states and territories in late March. National borders were incrementally closed to foreign nationals with all returned travellers required to self-isolate for 14 days in hotel quarantine from 28 March [2]. 


\section{FIGURE 1}

Surveillance data on (A) influenza and COVID-19 notifications and measures implemented, (B) ILI consultation rates by week and (C) total influenza admissions per week at national sentinel hospitals, Australia, as at 30 September 2020

\section{A. Influenza and COVID-19 notifications and measures implemented}

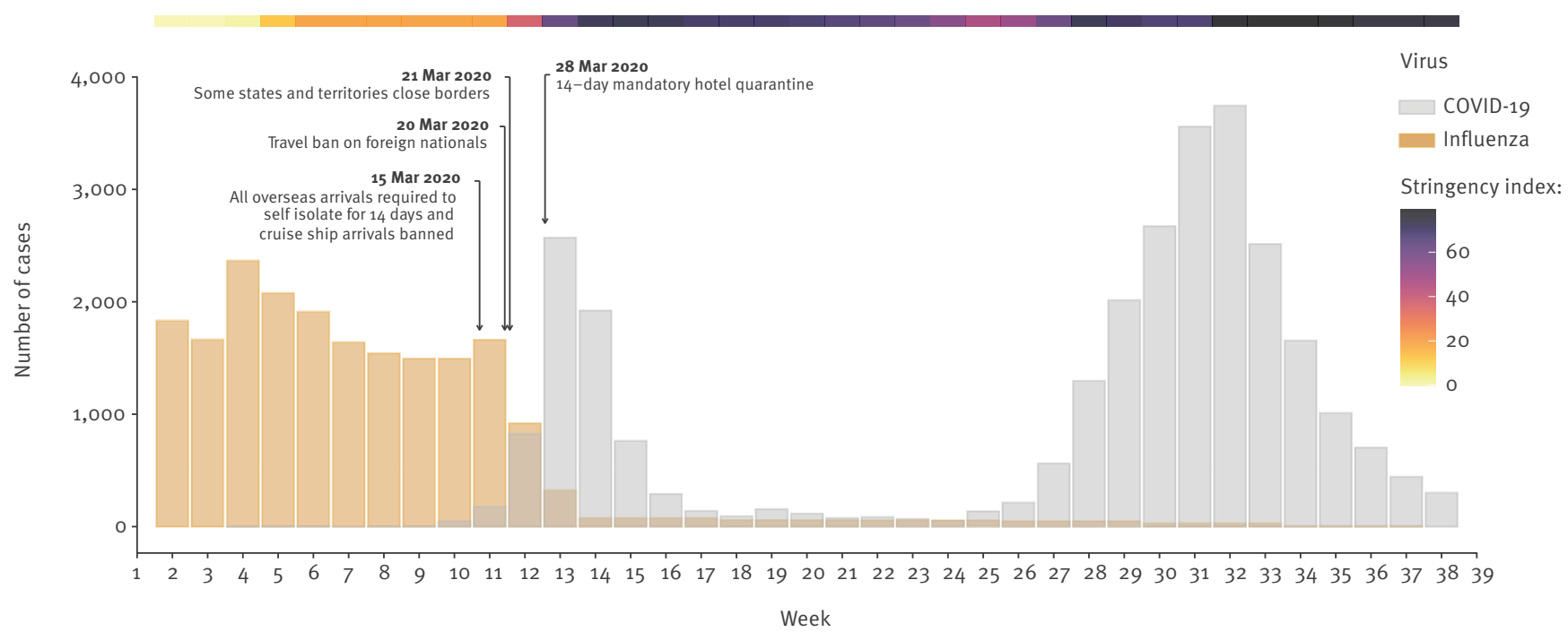

\section{B. ILI consultation rates by week}

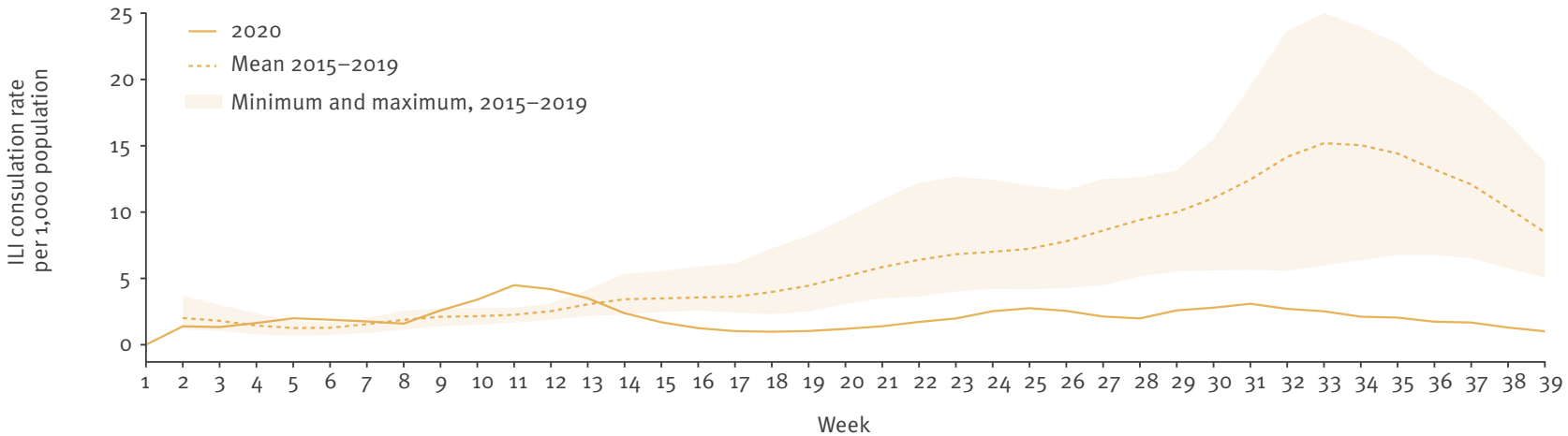

\section{Total influenza admissions at national sentinel hospitals}

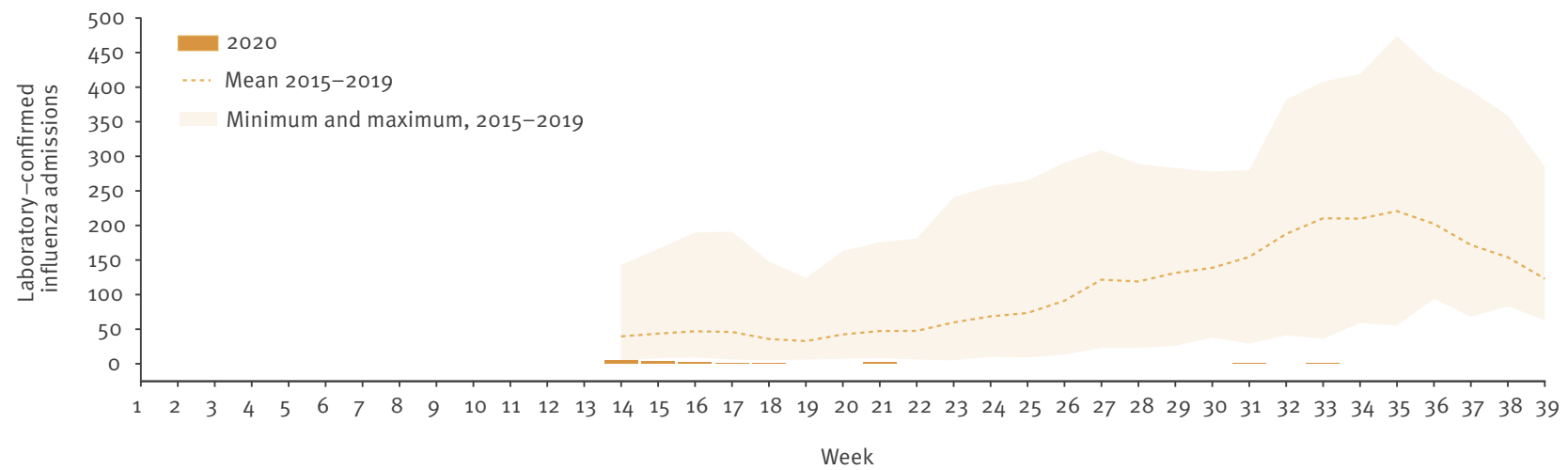

COVID-19: coronavirus disease; ILI: influenza-like illness.

Panel A: Influenza notifications are based on monthly notifications with weekly variation inferred from weekly counts for New South Wales. The Oxford Stringency Index indicates the severity of government restrictions [1]. Source: National Notifiable Diseases Surveillance System and www.covid19data.com.au.

Panel B: 3-week moving average. Source: Australian Sentinel Practices Research Network.

Panel C: Virological testing only detected one influenza virus since April. Source: Influenza Complications Alert Network. 
Influenza is notifiable in Australia and notifications averaged around 2,000 cases per week from January to March 2020, with a precipitous drop between mid-March (week 11) and mid-April (week 14), when travel and work restrictions were introduced (Figure $1 \mathrm{~A})$. Concurrently, COVID-19 notifications began to increase, peaking in April 2020 at levels comparable to the inter-seasonal influenza activity seen from January to February. Physical, but not travel, restrictions were somewhat relaxed from May to June for most of the country (Figure 1A [2];). A second COVID-19 epidemic was experienced in the state of Victoria only, in July 2020 , leading to the re-implementation of strict control measures and accounting for the vast majority of cases notified (94\%) from July to September. However, the subsequent relaxation of restrictions across most of the country has not been accompanied by increases in influenza notifications during the normal influenza season (approximately weeks 18-44).

\section{Sentinel surveillance}

In Australia, influenza-like illness (ILI) surveillance in primary care is conducted year-round to capture seasonal and inter-seasonal influenza patterns, as well as activity in the tropical northern region and infections in individuals following international travel [3]. ILI surveillance usually shows clear seasonality, with consultation rates highest in July and August (Figure 1B). However, following considerable influenza activity in 2019 [4], ILI consultation rates were well below average in 2020. ILI sentinel surveillance detected just one influenza and three respiratory syncytial virus (RSV) cases among 587 swabs tested from April to September.

National sentinel hospitalisation data (20 sites, FluCAN-PAEDS network [5]) reflects a similar steep decline in influenza-associated hospitalisations (Figure $1 C)$. There were also far fewer influenza-attributable deaths reported nationally (36 deaths from January to September 2020, compared with 812 deaths for the same period in 2019 [6]) and, since April, deaths attributed to respiratory causes have been below historical averages [7].

Consistency in primary care, hospitalisation and mortality data all suggest that these declines cannot be entirely attributed to changes in healthcare-seeking behaviour.

\section{Virological surveillance}

Real-time RT-PCR testing capacity in Australia considerably expanded in 2020 with the introduction of, and substantial demand for, SARS-CoV-2 testing. Initially, COVID-19 disrupted testing for non-SARS-CoV-2 respiratory viruses at some laboratories. However, even after public and private laboratories increased testing capacity for other respiratory viruses (by up to a threefold increase in some jurisdictions (data not shown)), influenza virus detections remained exceedingly low [6]. Data from New South Wales (NSW) and Western Australia (WA) showed major reductions in the weekly percentages of tests positive for either influenza viruses or RSV (Figure 2B-C). Bronchiolitis surveillance from NSW emergency departments corroborates this drastic decline in RSV cases (Figure 2A). In contrast, rhinovirus detections were well above average.

To enable total case finding for COVID-19, the threshold for respiratory virus testing has declined, with the broadening of case definitions and indications for testing, irrespective of the presence of symptoms. These minimal criteria may explain the very high percentage of detections positive for rhinovirus infection in NSW and WA since around week 22 (Figure 2). It is possible that these infections were detected in people who may not have met clinical criteria for testing in prior years. Alternatively, absent competition from influenza and RSV [8], rhinoviruses may be taking advantage of this niche. The drop in rhinovirus percentage-positivity around weeks 15-21 coincided with school holidays, 4-week school closures in NSW and voluntary withdrawal of children from school by many parents, underscoring the role of children in rhinovirus circulation [9].

\section{Discussion}

The very low numbers of influenza virus detections since April raises the possibility that some of the notified infections may be false positive. Among 10 influenza viruses $(6.7 \%$ of all notified by diagnostic laboratory testing) received in August and September at the WHO Collaborating Centre for Reference and Research on Influenza in Melbourne, none could be confirmed as influenza by real-time RT-PCR or culture. Given that the surveillance data suggested a prevalence $<0.05 \%$ [10], a test with $95 \%$ sensitivity and $99.99 \%$ specificity would still only be expected to have a positive predictive value of $82.6 \%$. This means that at least $15 \%$ of samples currently being diagnosed as influenza positive may be false positive.

Australia's annual influenza vaccination campaign commenced in April 2020. Vaccination, funded under the National Immunisation Program, was provided free of charge to many high-risk individuals [11,12] and all Australians were strongly encouraged to become vaccinated to alleviate any unnecessary burden on healthcare services should SARS-CoV-2 and influenza viruses co-circulate. Vaccine supply was sufficient to cover $70 \%$ of the eligible population and meet heightened demand in 2020 [13]. ILI surveillance data suggested uptake increased from $27 \%$ in 2019 to $45 \%$ in 2020. Online ILI surveillance data available through Flutracking [14] suggested that up to $93 \%$ of healthcare workers were vaccinated this year, an increase from $88 \%$ in 2019 . While it is possible that vaccination may have made a small contribution to reducing the spread of influenza, distinguishing the relative contribution of vaccination from that of physical distancing, movement and travel restrictions, hand hygiene and other measures is difficult. 


\section{FIGURE 2}

Selected surveillance data on (A) emergency department visit rates by week and weekly laboratory testing for respiratory viruses from (B) NSW and (C) WA, Australia, January-September 2020

\section{A. Emergency department visit rates by week}

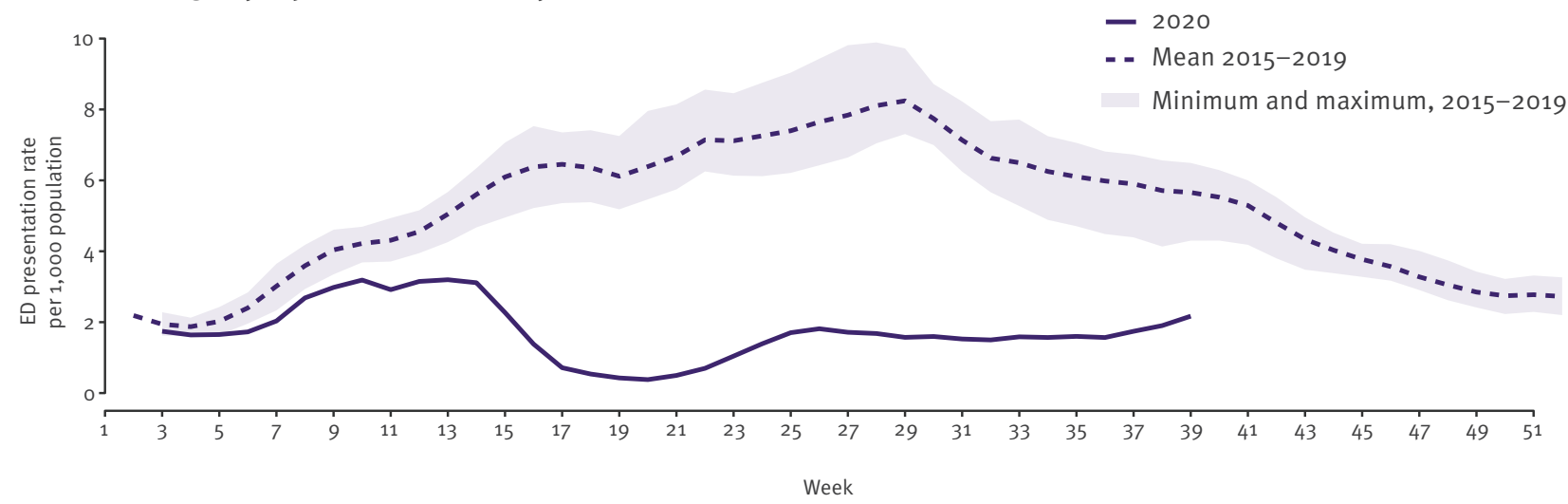

\section{B. Laboratory testing for respiratory viruses from NSW}

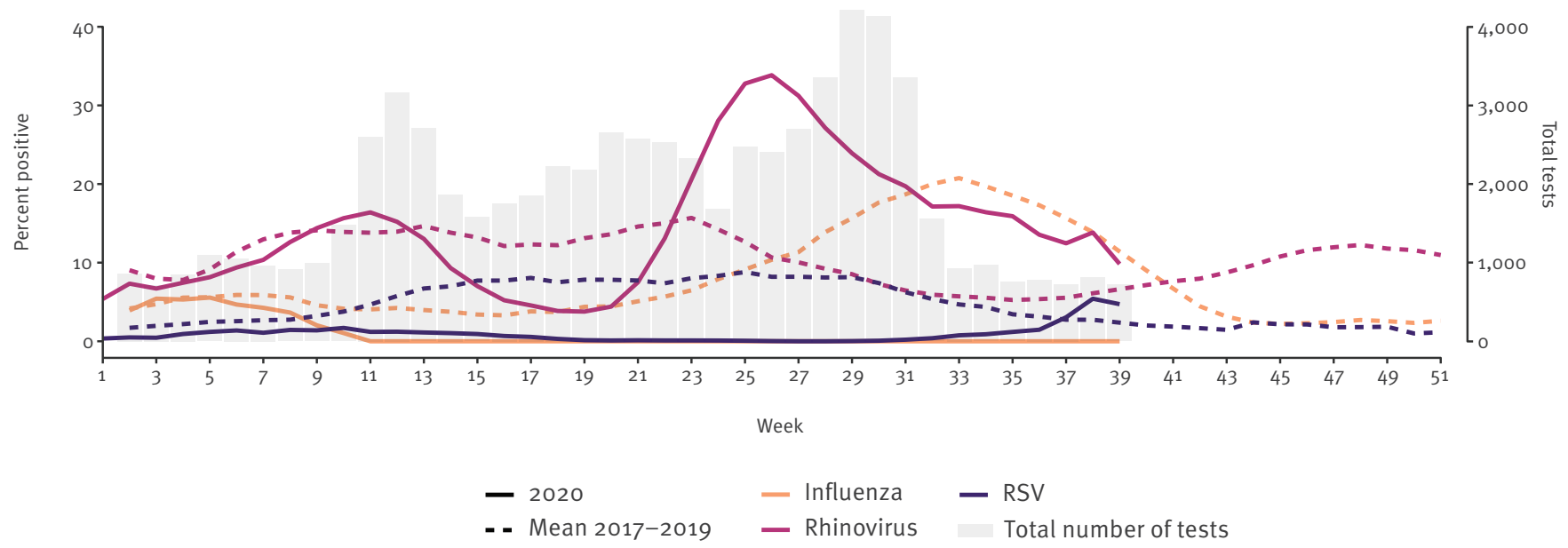

\section{Laboratory testing for respiratory viruses from WA}

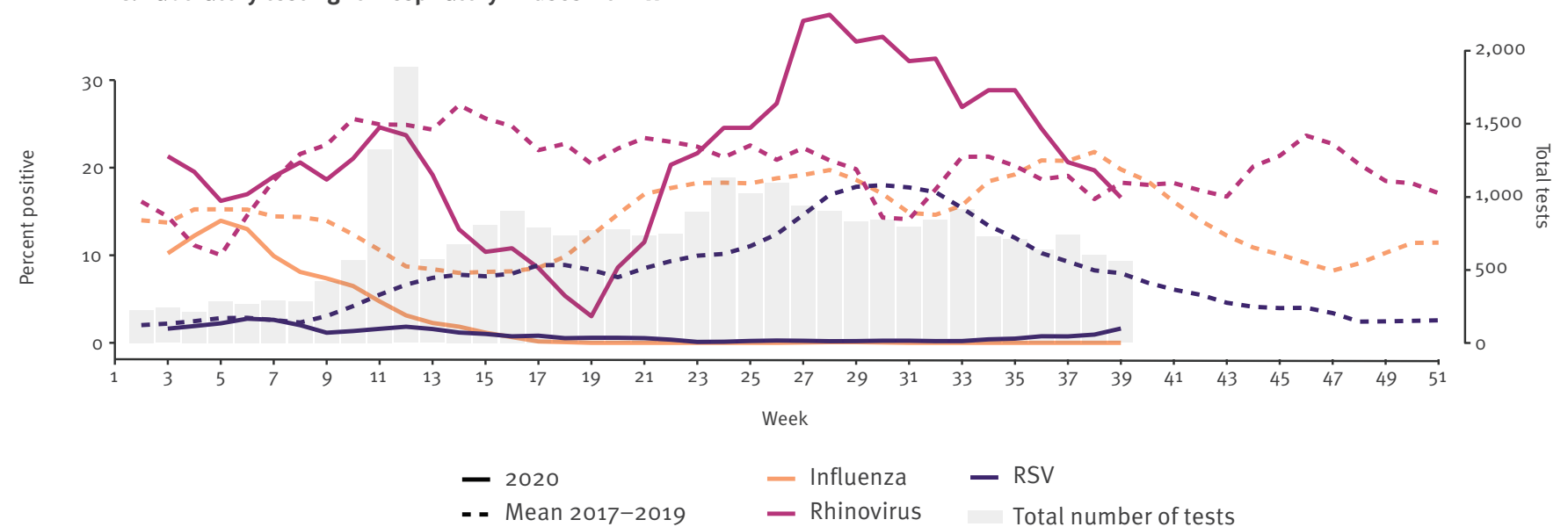

ED: emergency department; NSW: New South Wales; RSV: respiratory syncytial virus; WA: Western Australia.

Panel A: 3-week moving average for bronchiolitis, a clinical proxy for RSV. Source: Public Health Rapid, Emergency, Disease and Syndromic Surveillance (PHREDSS) system, Centre for Epidemiology and Evidence, NSW Ministry of Health.

Panel B: Respiratory viruses tested were influenza, RSV and rhinoviruses. Lines show the 3-week moving average for each virus. Grey bars show the total tests performed per week (right y-axis). Source: New South Wales Health Pathology.

Panel C: Respiratory viruses tested were influenza, RSV and rhinoviruses. Grey bars show the total tests performed per week (right y-axis). Source: PathWest Laboratory Medicine, Perth.

The state's capital, Perth, where most of the population resides, is ca $3,000 \mathrm{~km}$ from the NSW capital, Sydney, yet the patterns of virus percent-positive are very similar in the two cities. 
We have previously shown that influenza epidemics in major Australian cities are highly synchronous, with frequent co-circulation of viruses from genetic subgroups in cities up to $3,000 \mathrm{~km}$ apart, suggesting significant mixing of the national population [15]. The closure of inter-jurisdictional borders appears to have effectively limited this mixing. In addition, genomic data suggest that influenza virus circulation in Australia usually involves multiple introductions from the global influenza virus population [15]. The drastic reduction in international travel and the mandatory 14-day quarantine in hotels on entry-which is sufficiently long and stringent for respiratory infections to resolve-as well as low circulation of influenza globally [16], has limited potential new virus introductions.

Like Australian jurisdictions, European countries frequently observe coinciding influenza seasons [17] that are dependent on introductions from other regions [18]. As at mid-November 2020, European sentinel surveillance indicates extremely low levels of influenza virus activity [19]. The limited number of influenza viruses currently circulating may have minimal opportunities to seed seasonal epidemics in Europe, as they are likely to be severely constrained by the recent re-implementation of restrictions on movement and mixing within the continent, as well as heavily reduced air travel to Europe.

\section{Conclusions}

The COVID-19 pandemic and related mitigation strategies have exerted a strong impact on the circulation of influenza, RSV and other respiratory viruses. Globally, influenza viruses are in a severe bottleneck, complicating influenza vaccine decision-making for the upcoming seasons. Recent circulation of influenza $A\left(\mathrm{H}_{3} \mathrm{~N}_{2}\right)$ viruses in Cambodia and Bangladesh from July to September highlights the potential for the re-introduction of annual epidemics once global travel resumes. Recent increases in RSV activity in NSW and South Africa [20] suggest that RSV recirculation may precede that of influenza. It could be some time before global respiratory pathogen circulation returns to normal levels. In the meantime, the 2020 pandemic restrictions may substantially ameliorate the winter respiratory pathogen epidemics in 2021 and beyond. Compared with many European countries, the pandemic control response in Australia has comprised more stringent border control and stricter rules to limit social mixing. Nevertheless, interventions used to limit person-toperson transmission of SARS-CoV-2 are the same as those that would be recommended in an influenza pandemic and should therefore be expected to limit the spread of seasonal influenza as well. Low circulation of influenza virus is already apparent in Europe [19], but whether this continues remains to be seen.
${ }^{*}$ Erratum

On publication, the date period 2015-2019 was incorrectly written as 2015-2109 in the abstract. This mistake was corrected on 21 May 2021. We apologise for any inconvenience this typo may have caused.

\section{Acknowledgements}

Rapid Surveillance, Centre for Epidemiology, NSW Ministry of Health for provision of the PHREDSS system bronchiolitis emergency department data.

Funding statement: The WHO Collaborating Centre for Reference and Research on Influenza, WHO Collaborating Centre for Viral Hepatitis, the Australian Sentinel Practices Research Network, the Influenza Complications Alert Network and FluTracking are all supported by the Australian Government Department of Health. The opinions expressed in this paper are those of the authors, and do not necessarily represent the views of the Department.

\section{Conflict of interest}

None declared.

\section{Authors' contributions}

SGS and IB conceived the study, prepared the first draft and managed all revisions; SC manages Australian FluTracking data and provided estimates for vaccination coverage from that system; AC and KM contributed FluCAN data and provided input into to the development of the manuscript; MBNC and NS manage ASPREN and contributed data and vaccination coverage estimates and relevant text; DED, JK and JT provided virological data for New South Wales and contributed to the development of the manuscript; JM provided estimates for testing sensitivity and specificity and associated text; CMS and DS provided virological data for Western Australia and contributed to manuscript development.

\section{References}

1. Hale T, Atav T, Hallas L, Kira B, Phillips T, Petherick A, et al. Coronavirus government response tracker 2020. Oxford: University of Oxford; 2020. Available from: https://www.bsg.ox.ac.uk/research/research-projects/ coronavirus-government-response-tracker

2. COVID-19 National Incident Room. COVID-19 Australia: Epidemiology Report 25. Commun Dis Intell. 2020;44:1-35.

3. Sullivan SG, Pennington K, Raupach J, Franklin LJ, Bareja C, de Kluyver R. A Summary of Influenza Surveillance Systems in Australia, 2015. Canberra: Department of Health; 2015.

4. Barr IG, Deng YM, Grau ML, Han AX, Gilmour R, Irwin M, et al. Intense interseasonal influenza outbreaks, Australia, 2018/19. Euro Surveill. 2019;24(33). https://doi.org/10.2807/1560-7917. ES.2019.24.33.1900421 PMID: 31431210

5. Cheng AC, Holmes M, Dwyer DE, Senanayake S, Cooley L, Irving LB, et al. Influenza epidemiology in patients admitted to sentinel Australian hospitals in 2018: the Influenza Complications Alert Network (FluCAN). Commun Dis Intell (2018). 2019;43:43. https://doi.org/10.33321/cdi.2019.43.48 PMID: 31738866

6. Australian Government Department of Health. Australian Influenza Surveillance Report. No. 12, 2020, 07 September to 20 September 2020. Canberra: Australian Government Department of Health; 2020.

7. Australia Bureau of Statistics (ABS). Provisional deaths data for measuring changes in patterns of mortality during the COVID-19 pandemic and recovery period. Reference period Jan-Jun 2020. Canberra: ABS; 2020. Available from 
https://www.abs.gov.au/statistics/health/causes-death/ provisional-mortality-statistics/latest-release

8. Wu A, Mihaylova VT, Landry ML, Foxman EF. Interference between rhinovirus and influenza A virus: a clinical data analysis and experimental infection study. Lancet Microbe. 2020;1(6):e254-62. https://doi.org/10.1016/S26665247(20)30114-2 PMID: 33103132

9. Baguelin M, Flasche S, Camacho A, Demiris N, Miller E, Edmunds WJ. Assessing optimal target populations for influenza vaccination programmes: an evidence synthesis and modelling study. PLoS Med. 2013;10(10):e1001527. https://doi. org/10.1371/journal.pmed.1001527 PMID: 24115913

10. Australian Sentinel Practices Research Network (ASPREN). ASPREN Update No 19. 2020 - 7th September - 20th September 2020. Adelaide: ASPREN; 2020 Available from: https://aspren.dmac.adelaide.edu.au/ documents/20714/469323/ASPREN+Update+19+2020/ a9549954-12f7-4608-9df4-34b87e2eeaf3?version=1.0

11. Beard F, Hendry A, Macartney K. Influenza vaccination uptake in our most vulnerable groups: how well are we protecting them in 2019? Commun Dis Intell. 2018;2020:44.

12. Australian Government Department of Health. National Immunisation Program Schedule: Flu (influenza) vaccines 2020. Canberra: Australian Government Department of Health; 2020. Available from: https://www.health.gov.au/healthtopics/immunisation/immunisation-throughout-life/nationalimmunisation-program-schedule\#flu-influenza-vaccines

13. Therapeutic Goods Administration. TGA Laboratories testing report: Seasonal influenza vaccines - Batch release 2020. Canberra: Australian Government Department of Health; 2020. Available from: https://www.tga.gov.au/node/911783

14. FluTracking. Weekly Interim Report: Australia, Week Ending 04 October 2020. Newcastle: FluTracking; 2020. Available from: https://www.flutracking.net/Info/Report/202041/AU

15. Geoghegan JL, Saavedra AF, Duchêne S, Sullivan S, Barr I, Holmes EC. Continental synchronicity of human influenza virus epidemics despite climatic variation. PLoS Pathog. 2018;14(1):e1006780. https://doi.org/10.1371/journal. ppat.1006780 PMID: 29324895

16. Global Influenza Surveillance and Response System. Influenza Laboratory Surveillance Information - Global circulation of influenza viruses 2020. Geneva: World Health Organization; 2020. Available from: https://apps.who.int/flumart/ Default?ReportNo $=6$

17. Caini S, Alonso WJ, Séblain CEG, Schellevis F, Paget J. The spatiotemporal characteristics of influenza A and B in the WHO European Region: can one define influenza transmission zones in Europe? Euro Surveill. 2017;22(35):30606. https://doi. org/10.2807/1560-7917.ES.2017.22.35.30606 PMID: 28877844

18. Bedford T, Riley S, Barr IG, Broor S, Chadha M, Cox NJ, et al. Global circulation patterns of seasonal influenza viruses vary with antigenic drift. Nature. 2015;523(7559):217-20. https:// doi.org/10.1038/nature14460 PMID: 26053121

19. European Centre for Disease Prevention and Control (ECDC). Communicable Diseases Threats Report Week 46, 8-14 November 2020. Stockholm: ECDC; 2020. Available from: https://www.ecdc.europa.eu/en/publications-data/ communicable-disease-threats-report-8-14-november-2020week-46

20. Cohen C. Influenza-SARS-CoV-2 co-circulation: lessons from the Southern hemisphere: the South African Experience. ECCVID: ESCMID Conference on Coronavirus Disease; 23-25 September 2020, online.

\section{License, supplementary material and copyright}

This is an open-access article distributed under the terms of the Creative Commons Attribution (CC BY 4.0) Licence. You may share and adapt the material, but must give appropriate credit to the source, provide a link to the licence and indicate if changes were made.

Any supplementary material referenced in the article can be found in the online version.

This article is copyright of the authors or their affiliated institutions, 2020. 